\title{
A Real-Life, International, Observational Study Demonstrating The Efficacy Of A Cosmetic Emulsion In The Supportive Care Of Mild-To-Moderate Facial Acne
}

This article was published in the following Dove Press journal:

Clinical, Cosmetic and Investigational Dermatology

\author{
Vincenzo Bettoli (ID) \\ Christine Coutanceau (D) ${ }^{2}$ \\ Victor Georgescu ${ }^{2}$ \\ 'Department of Medical Sciences, \\ Operational Unit of Dermatology, \\ Azienda Ospedaliera - University of \\ Ferrara, Ferrara, Italy; ${ }^{2}$ Laboratoires \\ Dermatologiques AVENE, Pierre Fabre \\ Dermo-Cosmétique, Lavaur, France
}

\begin{abstract}
Purpose: Cosmetic emulsions are increasingly being used as supportive care products to alleviate the severe side effects and improve the clinical outcomes associated with conventional acne treatments. The objectives of this study were to determine, in a real-life setting, the global effectiveness of an emulsion with antiseborrheic, keratolytic and anti-Propionibacterium acnes activities as an adjunct to anti-acne therapy, and to evaluate the effect of the product on acne severity, noninflammatory and inflammatory lesions, hyperseborrhea, skin irritation and patient quality of life (QoL). Tolerance of the product was also assessed.
\end{abstract}

Patients and methods: This international observational study involved 3960 patients aged 12 years and over with mild-to-moderate acne. The conventional acne prescriptions for these patients were either initiated at inclusion or were reviewed. Reviewed prescriptions may have been left unchanged, switched, or an addition made to ongoing treatment. At inclusion, participants were instructed to apply the cosmetic product daily for 2-3 months in combination with their medical acne treatment.

Results: After 2 to 3 months of use, the combined therapy resulted in moderate-to-large improvements in acne in over $75 \%$ of the patients whose acne prescriptions were left unchanged $(n=859)$. The combination treatment led to an improvement in acne severity $(-38.3 \%$ change in the mean investigator's global assessment score; $\mathrm{p}<0.0001)$ and QoL $(-38.3 \%$ change in the mean Cardiff acne disability index score; $\mathrm{p}<0.0001)$, and reduced noninflammatory and superficial inflammatory lesions $(-35.3 \%$ and $-47.0 \%$, respectively; $\mathrm{p}<0.0001)$, hyperseborrhoea $(-40.6 \% ; \mathrm{p}<0.0001)$ and skin irritation $(-37.2 \% ; \mathrm{p}<0.0001)$. Tolerance was very good.

Conclusion: Use of the cosmetic emulsion combined with conventional acne therapy significantly improved clinical outcomes and patient QoL, and was very well tolerated. The combined therapy may have improved patient satisfaction, leading to improved patient adherence and higher rates of therapeutic success.

Keywords: acne vulgaris, hyperseborrhoea, Propionibacterium acnes, acne treatment, supportive care, dermocosmetic product

\section{Introduction}

Acne vulgaris (commonly referred to as acne) is a very common dermatological condition. It is ranked as the eighth most prevalent disease worldwide and affects an estimated $9.4 \%$ of the global population. ${ }^{1}$ Although it mostly affects adolescents, ${ }^{2}$ acne occurs in patients of all ages, with many individuals experiencing acne during early and
Correspondence: Christine Coutance Pierre Fabre Dermo-Cosmétique, Les Cauquillous, Lavaur Cedex 81 506,

France

Tel +33563589652

Fax +33 563588667

Email christine.coutanceau@pierre-

fabre.com 
even late adulthood. ${ }^{3,4}$ Acne is far from being a uniquely cosmetic or superficial condition and can have an emotional impact on patients with long-lasting psychosocial effects. Scarring and disfigurement can have a highly detrimental effect on quality of life (QoL) and have an impact on selfesteem, contributing to depression and anxiety. ${ }^{5-9}$

Acne is a chronic inflammatory disorder of the pilosebaceous unit that typically manifests with comedones, papules, pustules, nodules and scars. Androgen-stimulated excess sebum production, ${ }^{10}$ follicular hyperkeratinization, ${ }^{11}$ microbial colonization with the Gram-positive anaerobic bacterium Propionibacterium acnes $(P \text {. acnes })^{11,12}$ and inflammation ${ }^{13-15}$ are key elements involved in the complex pathophysiology of acne. Although the exact underlying mechanisms have not been fully elucidated, an improved understanding of acne pathogenesis ${ }^{16,17}$ has led to the development of new therapeutic modalities for better disease management. Topical retinoids, benzoyl peroxide (BPO) and systemic antibiotics are currently the first-line treatments for mild-to-moderate acne. ${ }^{18}$ Although highly effective, topical treatments affect skin barrier integrity and are often associated with side effects such as dryness, irritation, itching and redness. These adverse events are usually temporary and mild. However, they can occasionally be severe or even intolerable, causing a great deal of discomfort to the patient and severely hampering already low levels of compliance with therapy. ${ }^{19,20}$ These low levels of compliance compromise treatment efficacy and have an impact on clinical outcomes. In addition, antibiotic resistance ${ }^{19}$ is a growing cause for concern and represents a major obstacle in acne management.

Scientific advances in our understanding of the complex mechanisms driving acne pathophysiology have led to the development of cosmetic products for use as beneficial adjuncts and complementary treatments to modern acne therapy. ${ }^{21-24}$ These products improve outcomes by helping to minimize often intolerable side effects, increasing the levels of compliance to treatment, ${ }^{20}$ supporting treatment in periods of remission, and improving patient QoL. ${ }^{22}$ In the past few years, only a handful of studies have investigated the effects of cosmetic adjunctive therapy in patients receiving acne treatment. ${ }^{25-28}$

The primary objective of this international observational study was to evaluate, in a real-life setting, the effectiveness of a cosmetic product when it was used as a supportive therapy, alongside conventional topical or systemic anti-acne treatments, by a large population of patients with mild-to-moderate facial acne. The product contained three active ingredients: an antiseborrheic agent, a keratolytic agent and an anti-P. acnes agent. It was freely prescribed by the investigator for use over a period of 2 to 3 months. The secondary objectives were to evaluate the effect of the treatment on the severity of the acne, noninflammatory and inflammatory lesions, hyperseborrhea and skin irritation, and on patient QoL. Tolerance to the combined therapy was also investigated.

\section{Subjects And Methods \\ Study Design And Subjects}

This study was carried out in 11 countries (Belgium, People's Republic of China [Hong-Kong], France, Germany, Italy, Mexico, Poland, Portugal, Romania, Russia and Spain), in accordance with applicable regulatory requirements in each country, from March 2015 to November 2016. This was an observational real-life Phase IV study conducted to assess the safety, tolerability and effectiveness of marketed medicines or cosmetics in clinical everyday practice, i.e., in a naturalistic setting where choice of therapy was consistent with approved prescribing information and in line with the usual everyday practice of the physician at his/her office. Other aspects of patient care, including clinical examinations, laboratory investigations, the use of instrumentation, and other invasive and noninvasive procedures were also in consonance with everyday practice. The drug/cosmetic was prescribed by the practitioner him/herself, as per his/her routine practice per label, and there was no systematic assignment of treatment. There was no invasive examination.

In France specifically, when an intervention is "devoid of risks and performed in the framework of usual everyday practice" (research called "research involving the human person Category III"), the law is as follows (Loi Jardé $n^{\circ} 2012-$ 300 of the 5 March 2012, modified by order $n^{\circ} 2016-800$ of the 16 June 2016): the patient must be informed and should not be opposed to his/her inclusion in the study and to the collection of data, but a signed informed consent is not necessary. Patients were therefore personally informed of their rights with regard to the processing of their personal data through an information leaflet translated into their native language, in accordance with the Regulation (EU) 2016/679 of the European Parliament and of the Council of 27 April 2016 on the protection of natural persons.

Patients in the age groups 12-18 years, 18-25 years, and those aged 25 years or older, with mild-to-moderate facial acne corresponding to a score of two to three on the 
investigator's global assessment (IGA) scale ${ }^{29}$ (i.e., easily recognizable acne involving approximately half of the face, with comedones, papules and pustules, and possibly one nodule), with a minimum of ten noninflammatory and five inflammatory lesions on the whole face (except the nasal pyramid), who were not being treated by systemic isotretinoin and who were prescribed the test product in addition to a medical topical or systemic anti-acne treatment were enrolled in the study. Noninclusion criteria were a known allergy to one or more components of the study product; excessive exposure to sunlight or ultraviolet rays within the previous month; initiation of contraceptive treatment within the past 3 months; clinically and laboratory demonstrated acne related to a clearly hyperandrogenic condition; a cutaneous pathology involving the studied zone with clinical significance and a potentially negative impact on the study results; any chronic systemic disorder or acute pathology that could interfere with the protocol, or the presentation of any condition for which the treatment could interfere with the protocol; and any other condition or treatment which, according to the investigator's judgement, would put the subject at undue risk or may interfere with the evaluation of the study results.

\section{Study Product}

The study product, designed for acne-prone skin, was a hypoallergenic cosmetic emulsion (Cleanance EXPERT, Eau Thermale Avène, France) with a physiological pH, consisting of patented highly specific anti-acne active ingredients: an antiseborrheic (monolaurin), a keratolytic agent $\left(\mathrm{X}-\mathrm{Pressin}{ }^{\mathrm{TM}}\right)$ and an agent with anti-inflammatory and anti-P. acnes activities (Diolényl ${ }^{\circledR}$, Patent No. WO2010072738A1). Participants were instructed to apply a small quantity of the product in the morning and/or evening, to the entire, freshly cleaned face. In case of a concomitant application of a medical topical anti-acne treatment, the study product had to be applied after complete absorption of the topical drug.

\section{Study Procedure}

At the inclusion visit, the investigator reviewed any current medical anti-acne treatments and was free to prescribe, or continue to prescribe, the medical anti-acne therapy of their choice in accordance with current guidelines. $^{30}$ The investigator was also free to provide recommendations (for example on the use of noncomedogenic makeup products, application of sunscreen in case of sun exposure and use of a cleansing product twice a day) according to their usual clinical practice without any specific instructions from the sponsor. Thus, included patients could have had any of the following treatment scenarios: 1) a medical anti-acne treatment initiated;2) their medical anti-acne treatment unchanged, with only the test cosmetic product added; 3) their medical treatment switched, for example from an antibiotic to BPO; or 4) a new medication added to their ongoing treatment, for example a topical therapy complemented with an oral treatment. Investigators were allowed to enroll a maximum of five patients per center. All participants also received a prescription for the study product as an adjunct treatment to their medical anti-acne therapy. Demographic data, acne history and medical anti-acne treatments at the moment of inclusion were recorded. Skin type and phototype were determined. Epidemiological data on the body sites affected by acne, predominant type of facial acne (noninflammatory, mixed or superficial inflammatory) and presence of secondary lesions (atrophic and hypertrophic scars, and pigmented macules) were also compiled. A follow-up visit was conducted, by the same investigator who carried out the inclusion assessments, after 2 to 3 months of product use (56-84 days). This visit was part of the systematic follow-up of the patient, by the dermatologist usually responsible for their management. No constraints were associated with this study and no additional invasive or specific examinations were carried out.

\section{Clinical Evaluations Primary Effectiveness Criterion}

The global effectiveness of the medical anti-acne treatments in combination with the cosmetic product was evaluated by measuring changes in facial acne at follow-up compared to inclusion according to a 6-point scale: 1: total disappearance of acne; 2: large improvement; 3: moderate improvement; 4: slight improvement; 5: no change; 6: aggravation. Given the large size of the cohort and the real-life setting used in this study, this scale was developed to allow direct evaluation of any overall changes in facial acne during the study period.

\section{Secondary Effectiveness Criteria}

The severity of facial acne was quantified at inclusion and at follow-up using the IGA scale ${ }^{29}$ from 0 (clear), 1 (almost clear), 2 (light), 3 (moderate) to 4 (severe) and the percentage change in the IGA score was calculated. A decrease in the score indicated improvement. 
The severity of objective clinical symptoms - such as noninflammatory and inflammatory lesions, hyperseborrhea and skin irritation - was graded by the physician at inclusion and follow-up using a 4-point scale from 0 (absent) to 3 (severe). The percentage change in the score was calculated between inclusion and follow-up. A decrease indicated improvement.

The impact of the skin condition on patient QoL was measured at inclusion and follow-up using the Cardiff acne disability index (CADI) ${ }^{31}$ patient questionnaire. Each answer was scored using a 4-point scale of decreasing severity from 3 (severe disability) to 0 (absence of disability). The overall CADI score was calculated by summing the score of each answer and ranged from 0 to 15. The higher the score, the more QoL was impaired.

\section{Tolerance}

Clinical tolerance was assessed by the dermatologist and the patient at follow-up using a 4-point scale (Table 1). Adverse events and serious adverse events were reported and documented throughout the study.

\section{Statistical Analysis}

All analyses were carried out using SAS software, version 9.2 (SAS Institute Inc., Cary, NC, USA). Analyses were conducted on data from three patient populations or subpopulations: all patients for whom epidemiological data were available (whole study population), patients for whom inclusion and follow-up visit data were available (per protocol [PP] population), and patients whose medical anti-acne treatment had not been changed at the inclusion visit (subpopulation

Table I Tolerance Evaluation Scale

\begin{tabular}{|l|l|l|}
\hline Score & Definition & Description \\
\hline 0 & Poor & $\begin{array}{l}\text { Functional and/or objective symptoms that } \\
\text { lead to discontinuation of product use }\end{array}$ \\
\hline 1 & Moderate & $\begin{array}{l}\text { Pronounced or persistent functional } \\
\text { discomfort symptoms or objective } \\
\text { symptoms that do not lead to } \\
\text { discontinuation of product use }\end{array}$ \\
\hline 2 & Good & $\begin{array}{l}\text { Mild transient functional symptoms that do } \\
\text { not lead to discontinuation of product use } \\
\text { and no objective symptoms upon } \\
\text { examination }\end{array}$ \\
\hline 3 & Very good & $\begin{array}{l}\text { No functional discomfort symptoms and no } \\
\text { objective symptoms upon examination }\end{array}$ \\
\hline
\end{tabular}

analysis). Epidemiological, demographic and acne characteristics at inclusion were analyzed for the whole population, tolerance was analyzed using data from the PP population, and the primary and secondary efficacy outcomes were analyzed using data from the PP population and the subpopulation. Indeed, due to the observational design of the study, the subpopulation of patients whose medical anti-acne treatment remained unchanged at inclusion was the only group in which the specific effectiveness of the cosmetic product could be evaluated by before-and-after intragroup comparisons. Missing data were not replaced and were treated as such in the statistical analysis. The numbers and percentages of patients were calculated for all variables. Quantitative variables were expressed as the mean \pm standard deviation. Median, min-max values and 95\% CIs were calculated when appropriate. Differences in severity scores between inclusion and follow-up, and percentage changes were analyzed using the Wilcoxon matchedpairs signed-rank test. A paired Student's $t$-test was used for CADI scores. Statistical significance was set at $\mathrm{p}<0.05$.

\section{Results}

\section{Baseline Demographics And Clinical Characteristics}

Participant flow through the study is shown in Figure 1. A total of 3960 patients were included in the study. Epidemiological data were available for 3955 of these patients and 3746 patients were included in the PP population. In the whole study population, 1966 patients (50.9\%) had their medical anti-acne treatment initiated at inclusion, 891 patients $(23.1 \%)$ had their medical treatment left unchanged at inclusion, 628 patients (16.2\%) were switched to another treatment and 380 patients (9.8\%) had another drug added to their treatment. Thirty-two of the patients whose medical anti-acne treatment remained unchanged at inclusion were lost to follow-up; the remaining 859 patients formed the subpopulation.

The average duration between visits was 61.7 days. The demographic and clinical characteristics of the whole study population are shown in Tables 2 and 3, respectively.

At inclusion, $89.1 \%(n=756)$ of the subpopulation of patients whose medical anti-acne treatment was left unchanged and $89.4 \%(n=3296)$ of the PP population had mild-to-moderate acne (Figure 2). There were no differences in the severity of facial acne, as indicated by the 


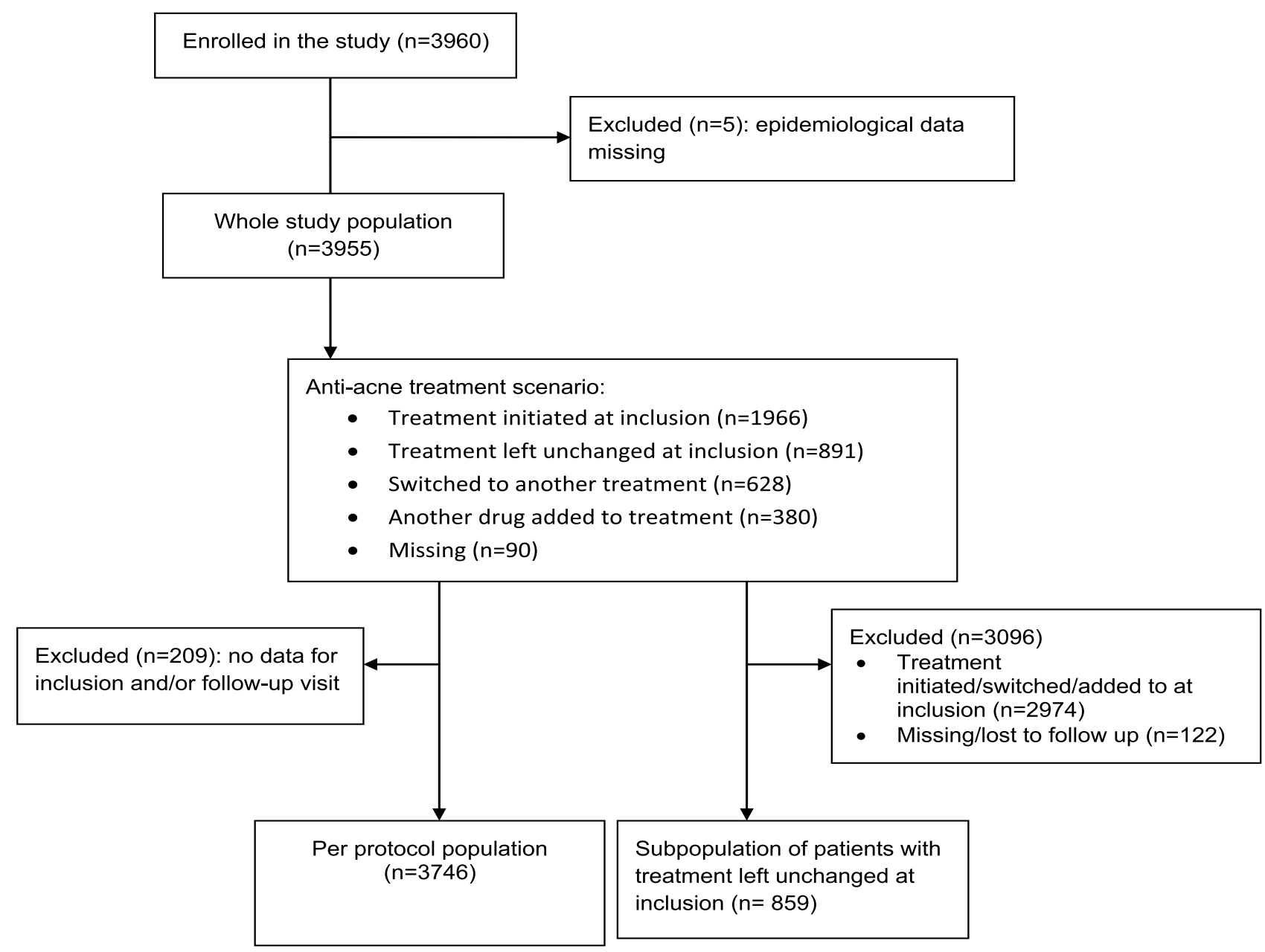

Figure I Participant flow through the study.

IGA scores, or in objective clinical symptoms between the two populations at inclusion (Table 4).

\section{Effectiveness Evaluations}

The results of the effectiveness analyses carried out on the PP population and those carried out on the subpopulation of patients whose medical anti-acne treatment remained unchanged at inclusion are presented in Figures 2 and 3, and in Tables 4 and 5. However, as the results obtained for the PP population for all evaluation criteria were similar to those obtained for the subpopulation, only the results for the subpopulation are described in this section.

\section{Primary Efficacy Criterion}

At the end of treatment, a large or moderate improvement of facial acne was recorded for $76.6 \%(n=653)$ of the patients whose medical anti-acne treatment remained unchanged at inclusion. Acne had totally disappeared in $7.9 \%(n=67)$ of these patients (Figure 3$)$.

\section{Secondary Efficacy Criteria Severity Of Facial Acne}

In the group whose medical treatment remained unchanged, the number of patients cleared and almost cleared of facial acne markedly increased from $9.0 \%(\mathrm{n}=77)$ at inclusion to $52.8 \%(\mathrm{n}=447)$ at the end of the 2- to 3-month study period, whereas the number of patients with moderate acne profoundly decreased from $38.9 \%(n=330)$ to $7.0 \% \quad(n=59)$ (Figure 2). The mean severity of acne also significantly improved, as shown by a $38.3 \%$ decrease in the average IGA score (difference in score: $-0.90 \pm 0.75, \mathrm{p}<0.0001$ ) (Table 4).

\section{Noninflammatory And Superficial Inflammatory Lesions}

A $35.3 \%$ and $47.0 \%$ reduction in the severity score for noninflammatory and inflammatory lesions, respectively, was recorded between inclusion and the end of the study period in the patients whose medical anti-acne treatment remained unchanged (differences in score: $-0.61 \pm 0.69, \mathrm{p}<0.0001$ and $-0.76 \pm 0.69, \mathrm{p}<0.0001$, respectively) (Table 4 ). 
Table 2 Demographics And Skin Characteristics At Inclusion In The Whole Study Population

\begin{tabular}{|c|c|}
\hline Parameter & $\begin{array}{l}\text { Whole Study Population At } \\
\text { Inclusion }(\mathrm{N}=3955)\end{array}$ \\
\hline Gender, n (\%) & $N=3919$ \\
\hline Male & $1337(34.1)$ \\
\hline Female & $2582(65.9)$ \\
\hline Age, years & $N=3922$ \\
\hline Mean $\pm S D$ & $18.4 \pm 4.1$ \\
\hline Median (min-max) & $18(11-49)$ \\
\hline Age groups, n (\%) & $N=3955$ \\
\hline $12-18$ years & $1905(48.2)$ \\
\hline $18-25$ years & $1707(43.2)$ \\
\hline$\geq 25$ years & $343(8.7)$ \\
\hline Skin characteristics, n (\%) ${ }^{\mathbf{a}}$ & $N=3680$ \\
\hline Mixed & $2437(66.2)$ \\
\hline Dry & $156(4.2)$ \\
\hline Oily & $1087(29.5)$ \\
\hline Skin phototype, $\mathbf{n}(\%)^{\mathbf{b}}$ & $N=3575$ \\
\hline I & $99(2.8)$ \\
\hline II & I324 (37.0) \\
\hline III & I 705 (47.7) \\
\hline IV & 396 (II.I) \\
\hline v & $36(1.0)$ \\
\hline VI & I5 (0.4) \\
\hline
\end{tabular}

Notes: ${ }^{a}$ Skin characteristics were described according to the investigator's clinical evaluation. 'Skin phototypes were defined according to the Fitzpatrick scale.

\section{Hyperseborrhea}

A $40.6 \%$ reduction in the severity score for hyperseborrhea was recorded between inclusion and the end of the study period in the patients whose medical anti-acne treatment remained unchanged (difference in score: -0.64 $\pm 0.69, \mathrm{p}<0.0001$ ) (Table 4).

\section{Skin Irritation}

A $37.2 \%$ decrease in the severity score for skin irritation was found between inclusion and the end of the study period in the patients whose medical treatment remained unchanged (difference in score: $-0.46 \pm 0.72, \mathrm{p}<0.0001$ ) (Table 4).

\section{Quality Of Life}

An improvement in QoL, as shown by the 38.3\% reduction in the global CADI score (difference in score: -2.32 $\pm 2.68, \mathrm{p}<0.0001$ ), was recorded between inclusion and the end of the study period in the patients whose medical antiacne treatment remained unchanged (Table 5).

\section{Tolerance}

Tolerance to the combined medical anti-acne and cosmetic treatment, rated by the clinicians and the patients themselves, was good to very good in around $95 \%(n=3533$ or $n=3470$, respectively) of the PP population, regardless of the medical anti-acne therapy being taken. Overall, 77\% $(n=2750)$ of the PP population considered that the test product had helped them to cope with their acne treatment and $89.8 \%(n=3257)$ wished to continue using it after the end of the study. Adverse events were reported by only $2.3 \%(n=83)$ of patients $(95 \%$ CI $[1.82 ; 2.80])$.

\section{Discussion}

In this international, observational, real-life study, the use of an adjunct cosmetic product for 2-3 months in association with conventional medical anti-acne therapy significantly improved the appearance and severity of mild-to-moderate acne. This combined therapy was globally very efficient. Moderate-to-large improvements in facial acne were observed in over $75 \%$ of the patients, and five times fewer patients were classed as having moderate acne by the end of the study period, both in the subpopulation of patients whose medical anti-acne treatment remained unchanged at inclusion and in the PP population. The combined treatment also led to a reduction in the number of noninflammatory and inflammatory lesions, hyperseborrhea and skin irritation. The product also led to improved patient QoL and was very well tolerated.

The predominance of females, the mean patient age of 18.4 years and the overall acne prevalence of $91.4 \%$ in patients aged between 12 and 24 years recorded in our population were in accordance with epidemiological data from previous studies. ${ }^{2,32}$ Less than $30 \%$ of our study population had a family history of acne, which is lower than the $62-75 \%$ reported in other studies. ${ }^{33,34}$ The treatments prescribed to the patients included in our study were mainly recommended first-line treatments for mild-tomoderate acne: topical retinoids, BPO, fixed combination retinoids and BPO, and systemic antibiotics. ${ }^{18,35}$ Some patients received oral zinc therapy, which has been widely used in the management of acne for decades. ${ }^{36}$

Although our study was centered around patients with mild-to-moderate acne at inclusion, we reported a highly significant improvement in the severity of acne in the PP population, with a $37.4 \%$ reduction in the IGA score. A similar and highly significant $38.3 \%$ decrease in the IGA score was observed in the subpopulation of patients who 
Table 3 Acne History And Clinical Characteristics At Inclusion In The Whole Study Population

\begin{tabular}{|c|c|c|}
\hline Parameter & & Whole Study Population $(\mathrm{N}=3955)$ \\
\hline \multicolumn{3}{|l|}{ Family history of acne, $\mathrm{n}$ (\%) } \\
\hline & Yes & $1124(29.2)$ \\
\hline Duration of acne prior to inclusion, years & $\begin{array}{l}\text { Mean } \pm S D \\
\text { Median (min-max) }\end{array}$ & $\begin{array}{l}3.3 \pm 3.3 \\
2(0-28)\end{array}$ \\
\hline \multicolumn{3}{|l|}{ Facial acne, $\mathbf{n}(\%)$} \\
\hline Sites affected & $\begin{array}{l}\text { Forehead } \\
\text { Cheeks } \\
\text { Mandibular region }\end{array}$ & $\begin{array}{l}3009(76.6) \\
2863(72.9) \\
2000(50.9)\end{array}$ \\
\hline Type of facial acne & $\begin{array}{l}\text { Mixed } \\
\text { Noninflammatory } \\
\text { Superficial inflammatory }\end{array}$ & $\begin{array}{l}2395(61.6) \\
668(17.2) \\
826(21.2)\end{array}$ \\
\hline Secondary lesions & $\begin{array}{l}\text { Presence of lesions } \\
\text { Atrophic scars } \\
\text { Hypertrophic scars } \\
\text { Hyperpigmented spots }\end{array}$ & $\begin{array}{l}1641(42.4) \\
720(47.4) \\
103(6.8) \\
1028(67.3)\end{array}$ \\
\hline \multicolumn{3}{|l|}{ Other body sites affected by acne, $n$ (\%) } \\
\hline & $\begin{array}{l}\text { Back } \\
\text { Neck } \\
\text { Shoulders } \\
\text { Chest }\end{array}$ & $\begin{array}{l}1254(35.2) \\
285(8.1) \\
754(21.2) \\
683(19.2)\end{array}$ \\
\hline \multicolumn{3}{|c|}{ History of acne treatment prior to inclusion, $n(\%)$} \\
\hline & Yes & $2704(70.2)$ \\
\hline \multicolumn{2}{|l|}{ Number of acne treatments prior to inclusion } & $N=2704$ \\
\hline & $\begin{array}{l}\text { Mean } \pm \text { SD } \\
\text { Median (min-max) }\end{array}$ & $\begin{array}{l}2.3 \pm 1.3 \\
2(1-9)\end{array}$ \\
\hline \multicolumn{3}{|l|}{ Type of prescription at inclusion, $n$ (\%) } \\
\hline & $\begin{array}{l}\text { Addition to an ongoing treatment } \\
\text { Initiation of treatment } \\
\text { Switched treatment } \\
\text { Unchanged treatment }\end{array}$ & $\begin{array}{l}380(9.8) \\
1966(50.9) \\
628(16.2) \\
891(23.1)\end{array}$ \\
\hline \multicolumn{3}{|l|}{ Acne treatment at inclusion ${ }^{a}, n(\%)$} \\
\hline Topical & $\begin{array}{l}\text { Retinoids } \\
\text { BPO } \\
\text { Fixed combination retinoids+BPO }\end{array}$ & $\begin{array}{l}763(20.2) \\
646(17.1) \\
643(17.0)\end{array}$ \\
\hline Systemic & $\begin{array}{l}\text { Zinc } \\
\text { Doxycycline }\end{array}$ & $\begin{array}{l}547(13.8) \\
67 \mid(17.7)\end{array}$ \\
\hline
\end{tabular}

Notes: ${ }^{a}$ Treatments prescribed to more than $10 \%$ of patients.

Abbreviation: BPO, benzoyl peroxide.

were already taking an anti-acne treatment that was not changed at inclusion. These results indicate that the improvements in acne severity in the PP population were not solely due to initiation or changes in the medical anti- acne therapy and that the effectiveness of the prescribed anti-acne regimens improved when they were used in combination with the adjunct cosmetic product. Similarly, the combined treatment led to highly significant improvements 


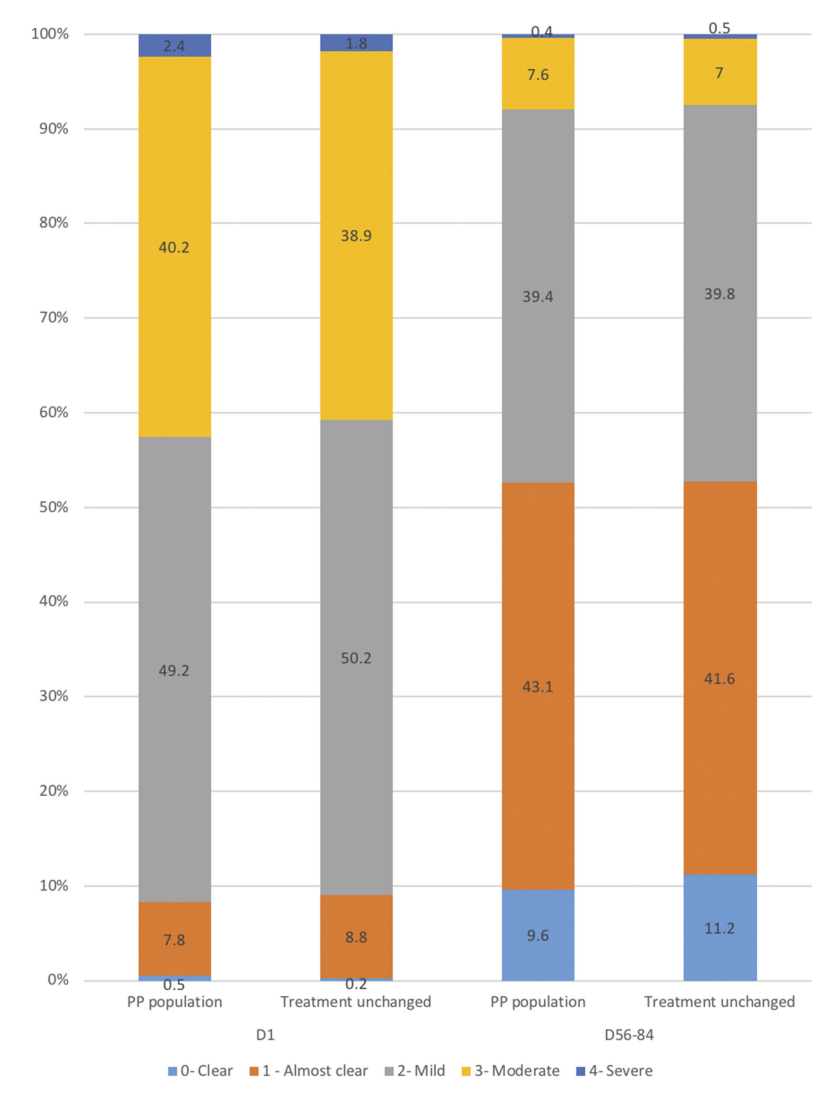

Figure 2 Severity of facial acne at inclusion and at follow-up after 2-3 months of use of the cosmetic product in combination with medical anti-acne therapy in the per protocol (PP) population ( $\mathrm{N}=3746)$ and in the subpopulation of patients whose medical antiacne treatment remained unchanged at inclusion $(\mathrm{N}=859)$. Severity was graded by the physician using the acne investigator's global assessment (IGA) scale from 0 (clear) to 4 (severe). The percentages of patients with each score are shown.

in the severity of noninflammatory and superficial inflammatory lesions, hyperseborrhea and skin irritation. The extent of improvement in these objective clinical symptoms was similar in the PP population and in the subpopulation of patients whose acne treatment remained unchanged at inclusion. These findings demonstrate that the adjunct cosmetic product was also effective at improving these objective clinical parameters.

Retinoids and BPO were prescribed at inclusion for some of the patients in our study. These treatments are often used in combination to combat the multiple pathogenic mechanisms that can be involved in the development of acne and therefore increase the likelihood of successful acne therapy; however, this combined therapy is often associated with an increased risk of side effects. Our cosmetic product contained active ingredients with antiseborrheic, keratolytic and anti-P. acnes properties. Our results suggest that the components of this product, when used in a complementary treatment together with conventional anti-acne therapies, might be highly beneficial for the global management of mild-to-moderate lesions. The benefits of this product can most likely be attributed to the complementary and/or synergistic mechanisms of action of the active ingredients, which effectively target excess production of sebum, follicular hyperkeratinization and microbial colonization with P. acnes, and enhance the tolerability of topical regimens.

In the present study, patients reported that acne had a moderate impact on their QoL at inclusion, with an average global CADI score of around five. Previous studies of patients with acne of similar severity to that noted in our study reported a markedly more impaired QoL, with CADI scores of seven, eight or above. ${ }^{8,22}$ Despite the moderate impact of acne on patient QoL in our study, we observed a highly significant improvement in QoL by the end of the study period. This improvement was observed both in the subpopulation of patients whose medical anti-acne treatment remained unchanged and in the PP population, with highly significant reductions in global CADI scores of more than one-third. These findings further confirm the effectiveness of the combined treatment.

Table 4 Acne Investigator's Global Assessment And Objective Clinical Symptom Severity Scores In The Per Protocol Population And In The Subpopulation Of Patients Whose Medical Anti-Acne Treatment Remained Unchanged At Inclusion

\begin{tabular}{|l|l|l|l|l|l|l|}
\hline \multirow{2}{*}{ Severity Scores Mean \pm SD } & \multicolumn{2}{|l|}{ PP Population (N=3746) } & \multicolumn{3}{l|}{ Patients Whose Treatment Remained Unchanged (N=859) } \\
\cline { 2 - 7 } & Inclusion & Follow-Up & \% Change & Inclusion & Follow-Up & \% Change \\
\hline IGA & $2.36 \pm 0.68$ & $1.46 \pm 0.78$ & $-37.4 * * * *$ & $2.33 \pm 0.67$ & $1.44 \pm 0.80$ & $-38.3 * * * *$ \\
Noninflammatory lesions & $1.55 \pm 0.65$ & $0.93 \pm 0.59$ & $-35.7 * * * *$ & $1.54 \pm 0.64$ & $0.93 \pm 0.63$ & $-35.3 * * * *$ \\
Superficial inflammatory lesions & $1.54 \pm 0.67$ & $0.73 \pm 0.61$ & $-50.0 * * * *$ & $1.50 \pm 0.65$ & $0.74 \pm 0.62$ & $-47.0 * * * *$ \\
Hyperseborrhea & $1.42 \pm 0.72$ & $0.72 \pm 0.62$ & $-43.8 * * * *$ & $1.38 \pm 0.72$ & $0.75 \pm 0.64$ & $-40.6 * * * *$ \\
Skin irritation & $0.75 \pm 0.77$ & $0.32 \pm 0.54$ & $-35.1 * * * *$ & $0.76 \pm 0.75$ & $0.29 \pm 0.53$ & $-37.2 * * * *$ \\
\hline
\end{tabular}

Notes: $* * * * 0.000 \mathrm{I}$ for the difference in severity scores between follow-up at the end of the 2-3 months of treatment and inclusion. Analyses were conducted using the Wilcoxon matched-pairs signed-rank test.

Abbreviations: PP, per protocol; IGA, investigator's global assessment. 


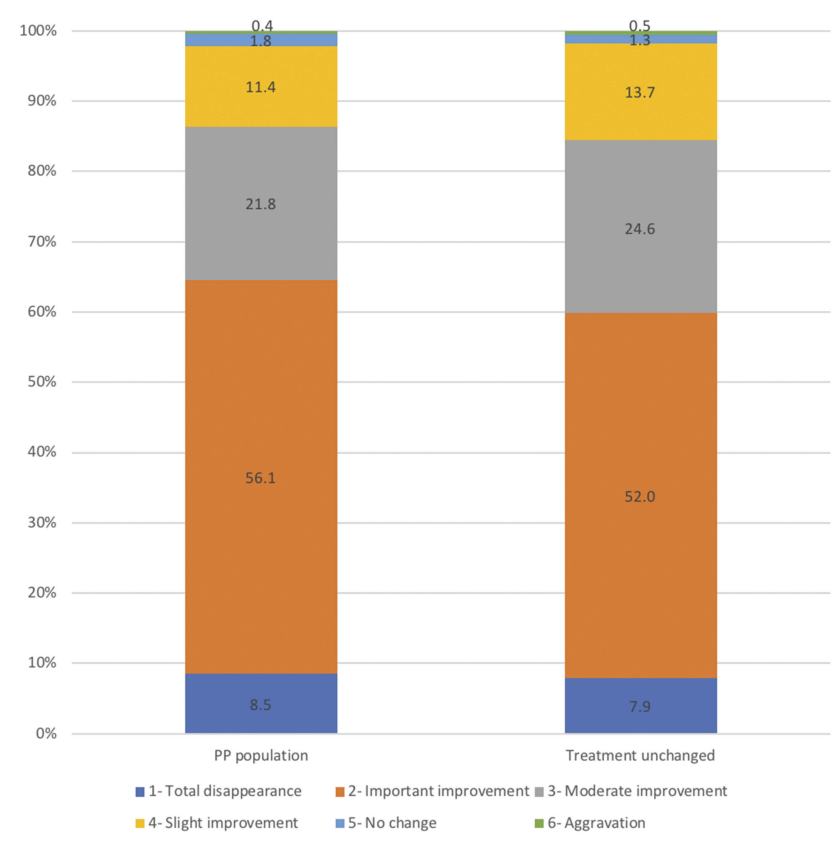

Figure 3 Global effectiveness at follow-up after 2-3 months of use of the cosmetic product in combination with medical anti-acne therapy in the per protocol (PP) population $(\mathrm{N}=3746)$ and in the subpopulation of patients whose medical anti-acne treatment remained unchanged at inclusion $(\mathrm{N}=859)$. Global effectiveness was measured by the physician with a 6-point scale from I (total disappearance) to 6 (aggravation). The percentages of patients with each score are shown.

Both the clinician and patient evaluations indicated that tolerance to the combined therapy after $2-3$ months of use was very good, and most patients wished to continue using the cosmetic product after the end of the study. Levels of satisfaction among patients with acne are an important issue given the substantial psychosocial impact of acne: positive emotional reactions may contribute to increased treatment adherence and more successful clinical outcomes.

The strength of our study was its noninterventional nature, which allowed a large international cohort of patients from 11 countries to be observed in a real-life setting over an extended time period. Each participating dermatologist was at liberty to prescribe the acne therapy of their choice without any recommendations from the sponsor, and patients followed their treatment plan using the product under everyday conditions. Although our conditions differed from those of a controlled clinical trial, during which patients often feel more compelled to adhere to their treatment and have a positive perception of the study, our study clearly demonstrated the global effectiveness of combining supportive treatment with the cosmetic product with conventional anti-acne therapy. However, the absence of any compliance assessments may be a potential limitation of this real-life study design. Indeed, use of the
Table 5 Global Cardiff Acne Disability Index (CADI) Score And CADI Score Category At Inclusion And At The End Of Treatment In The Per Protocol Population And In The Subpopulation Of Patients Whose Medical Anti-Acne Treatment Remained Unchanged At Inclusion

\begin{tabular}{|c|c|c|}
\hline Global CADI Score & Inclusion & Follow-Up \\
\hline \multicolumn{3}{|l|}{ PP population $(\mathrm{N}=3746)$} \\
\hline Mean \pm SD & $5.0 \pm 3.0$ & $2.7 \pm 2.4$ \\
\hline Median (min-max) & $5.0(0-15.0)$ & $2.0(0-13.0)$ \\
\hline$\%$ change & \multicolumn{2}{|l|}{$-39.4 * * * *$} \\
\hline \multicolumn{3}{|c|}{ Patients with treatment unchanged $(\mathrm{N}=859)$} \\
\hline Mean $\pm S D$ & $5.1 \pm 3.0$ & $2.7 \pm 2.2$ \\
\hline Median (min-max) & $5.0(0-15.0)$ & $3.0(0-12.0)$ \\
\hline$\%$ change & \multicolumn{2}{|l|}{$-38.3 * * * *$} \\
\hline \multicolumn{3}{|l|}{ CADI score category, $\mathrm{n}(\%)[95 \% \mathrm{Cl}]$} \\
\hline \multirow[t]{2}{*}{0 : Quality of life not affected by acne } & $186(5.0)$ & $829(22.2)$ \\
\hline & {$[4.3 ; 5.7]$} & {$[20.9 ; 23.5]$} \\
\hline \multirow{2}{*}{$\begin{array}{l}\text { I: Quality of life slightly affected } \\
\text { by acne }\end{array}$} & $2137(57.1)$ & $2485(66.5)$ \\
\hline & {$[55.5 ; 58.7]$} & {$[64.9 ; 68.0]$} \\
\hline \multirow{2}{*}{$\begin{array}{l}\text { 2: Quality of life moderately } \\
\text { affected by acne }\end{array}$} & $1258(33.6)$ & $407(10.9)$ \\
\hline & {$[32.1 ; 35.2]$} & {$[9.9 ; 11.9]$} \\
\hline \multirow{2}{*}{$\begin{array}{l}\text { 3: Quality of life severely affected } \\
\text { by acne }\end{array}$} & $159(4.2)$ & $17(0.4)$ \\
\hline & {$[3.7 ; 5.0]$} & {$[0.3 ; 0.7]$} \\
\hline
\end{tabular}

Notes: $* * * * \mathrm{P}<0.0001$ for the difference in global CADI score between follow-up at the end of the 2-3 months of treatment and inclusion. Analyses were carried out using the Student's $t$-test.

Abbreviation: CADI, Cardiff acne disability index.

cosmetic product may well have had an impact on compliance to conventional anti-acne regimens, with the reported effectiveness likely resulting from a combination of the direct action of the active ingredients and increased adherence to the medical anti-acne regimen as use of the product alleviated the side effects and the conventional medications became more tolerable. As this was an observational study, it was not possible to include a control group receiving a placebo in addition to the anti-acne therapy. As an alternative, we used a before-and-after design to evaluate the effectiveness of the study product in the subpopulation of patients whose medical anti-acne treatment remained unchanged at inclusion, allowing each patient to serve as their own control.

In conclusion, this international observational study demonstrated the benefits of using the cosmetic skin care product, Cleanance EXPERT, as an adjuvant care modality to improve clinical outcomes and minimize the harmful 
side effects of conventional treatment regimens for mildto-moderate acne. Tolerance to the combined therapy was very good, regardless of the prescribed anti-acne regimen. This good level of tolerance had a positive impact on patient QoL, which could, in turn, lead to increases in patient satisfaction, treatment adherence and levels of therapeutic success.

\section{Acknowledgments}

The authors thank all the participating investigators who enrolled patients in this study, as well as Emma Pilling and Marielle Romet, PhD, Synergy Pharm for providing medical writing assistance and English language editing on behalf of Laboratoires Dermatologiques Avène, Pierre Fabre DermoCosmétique. This work was supported by Laboratoires Dermatologiques Avène, Pierre Fabre Dermo-Cosmétique.

\section{Disclosure}

Dr Bettoli received fees from Laboratoires Dermatologiques Avène, Pierre Fabre Dermo-Cosmétique as scientific expert of the study, and Drs C Coutanceau and V Georgescu are employees of Laboratoires Dermatologiques Avène, Pierre Fabre Dermo-Cosmetique. The authors report no other conflicts of interest in this work.

\section{References}

1. Hollestein LM, Nijsten T. An insight into the global burden of skin diseases. J Invest Dermatol. 2014;134(6):1499-1501. doi:10.1038/ jid.2013.513

2. Ghodsi SZ, Orawa H, Zouboulis CC. Prevalence, severity, and severity risk factors of acne in high school pupils: a community-based study. J Invest Dermatol. 2009;129(9):2136-2141. doi:10.1038/ jid.2009.47

3. Collier CN, Harper JC, Cafardi JA, et al. The prevalence of acne in adults 20 years and older. $J$ Am Acad Dermatol. 2008;58(1):56-59. doi:10.1016/j.jaad.2007.06.045

4. Holzmann R, Shakery K. Postadolescent acne in females. Skin Pharmacol Physiol. 2014;27(Suppl 1):3-8. doi:10.1159/000354887

5. Halvorsen JA, Stern RS, Dalgard F, Thoresen M, Bjertness E, Lien L. Suicidal ideation, mental health problems, and social impairment are increased in adolescents with acne: a population-based study. $J$ Invest Dermatol. 2011;131(2):363-370. doi:10.1038/jid.2010.264

6. Misery L. Consequences of psychological distress in adolescents with acne. J Invest Dermatol. 2011;131(2):290-292. doi:10.1038/jid.2010.375

7. Hazarika N, Archana M. The psychosocial impact of acne vulgaris. Indian J Dermatol. 2016;61(5):515-520. doi:10.4103/0019-5154.190102

8. Zaraa I, Belghith I, Ben Alaya N, Trojjet S, Mokni M, Ben Osman A. Severity of acne and its impact on quality of life. Skinmed. 2013;11 (3):148-153.

9. Vilar GN, Santos LA, Sobral Filho JF. Quality of life, self-esteem and psychosocial factors in adolescents with acne vulgaris. An Bras Dermatol. 2015;90(5):622-629. doi:10.1590/abd1806-4841.201533726

10. Makrantonaki E, Ganceviciene R, Zouboulis C. An update on the role of the sebaceous gland in the pathogenesis of acne. Dermatoendocrinol. 2011;3(1):41-49. doi:10.4161/derm.3.1.13900
11. Jahns AC, Eilers H, Ganceviciene R, Alexeyev OA. Propionibacterium species and follicular keratinocyte activation in acneic and normal skin. Br J Dermatol. 2015;172(4):981-987. doi:10.1111/bjd.13436

12. Beylot C, Auffret N, Poli F, et al. Propionibacterium acnes: an update on its role in the pathogenesis of acne. J Eur Acad Dermatol Venereol. 2014;28(3):271-278. doi:10.1111/jdv.12224

13. Contassot E, French LE. New insights into acne pathogenesis: propionibacterium acnes activates the inflammasome. $J$ Invest Dermatol. 2014;134(2):310-313. doi:10.1038/jid.2013.505

14. Kim J. Review of the innate immune response in acne vulgaris: activation of Toll-like receptor 2 in acne triggers inflammatory cytokine responses. Dermatology. 2005;211(3):193-198. doi:10.1159/000087011

15. Taylor M, Gonzalez M, Porter R. Pathways to inflammation: acne pathophysiology. Eur J Dermatol. 2011;21(3):323-333. doi:10.1684/ ejd.2011.1357

16. Suh DH, Kwon HH. What's new in the physiopathology of acne? $\mathrm{Br} J$ Dermatol. 2015;172(Suppl 1):13-19. doi:10.1111/bjd.2015.172.issue-S1

17. Kircik LH. Advances in the understanding of the pathogenesis of inflammatory acne. J Drugs Dermatol. 2016;15(1 Suppl 1):s7-s10.

18. Zaenglein AL, Pathy AL, Schlosser BJ, et al. Guidelines of care for the management of acne vulgaris. J Am Acad Dermatol. 2016;74 (5):945-973.e933. doi:10.1016/j.jaad.2015.12.037

19. Humphrey S. Antibiotic resistance in acne treatment. Skin Therapy Lett. 2012;17(9):1-3.

20. Moradi Tuchayi S, Alexander TM, Nadkarni A, Feldman SR. Interventions to increase adherence to acne treatment. Patient Prefer Adherence. 2016;10:2091-2096. doi:10.2147/PPA.S117437

21. Guerrero D. [Dermo-cosmetic approach to acne by the dermatologist]. Ann Dermatol Venereol. 2010;137(Suppl 2):S76-S80. doi:10.1016/S0151-9638(10)70032-6

22. Araviiskaia E, Dreno B. The role of topical dermocosmetics in acne vulgaris. J Eur Acad Dermatol Venereol. 2016;30(6):926-935. doi:10.1111/jdv.13579

23. Dall'oglio F, Tedeschi A, Fabbrocini G, Veraldi S, Picardo M, Micali G. Cosmetics for acne: indications and recommendations for an evidencebased approach. G Ital Dermatol Venereol. 2015;150(1):1-11.

24. Goh CL, Noppakun N, Micali G, et al. Meeting the challenges of acne treatment in Asian patients: a review of the role of dermocosmetics as adjunctive therapy. J Cutan Aesthet Surg. 2016;9(2):85-92. doi:10.4103/0974-2077.184043

25. Bartenjev I, Oremovic L, Rogl Butina M, et al. Topical effectiveness of a cosmetic skincare treatment for acne-prone skin: a clinical study. Acta Dermatovenerol Alp Pannonica Adriat. 2011;20(2):55-62.

26. Trompezinski S, Weber S, Cadars B, et al. Assessment of a new biological complex efficacy on dysseborrhea, inflammation, and Propionibacterium acnes proliferation. Clin Cosmet Investig Dermatol. 2016;9:233-239. doi:10.2147/CCID.S110655

27. Saint-Jean M, Khammari A, Seite S, Moyal D, Dreno B. Characteristics of premenstrual acne flare-up and benefits of a dermocosmetic treatment: a double-blind randomised trial. Eur J Dermatol. 2017;27(2):144-149.

28. Polakova K, Fauger A, Sayag M, Jourdan E. A dermocosmetic containing bakuchiol, Ginkgo biloba extract and mannitol improves the efficacy of adapalene in patients with acne vulgaris: result from a controlled randomized trial. Clin Cosmet Investig Dermatol. 2015;8:187-191. doi:10.2147/CCID.S81691

29. Food and Drug Administration. Center for Drug Evaluation and Research. Guidance for Industry. Acne Vulgaris: Developing Drugs for treatment. In: Services. DoHaH; September 2005.

30. Nast A, Dreno B, Bettoli V, et al. European evidence-based (S3) guidelines for the treatment of acne. J Eur Acad Dermatol Venereol. 2012;26 (Suppl 1):1-29. doi:10.1111/j.1468-3083.2011.04374.x

31. Motley RJ, Finlay AY. Practical use of a disability index in the routine management of acne. Clin Exp Dermatol. 1992;17(1):1-3. doi:10.1111/j.1365-2230.1992.tb02521.x

32. Bhate K, Williams HC. Epidemiology of acne vulgaris. $\mathrm{Br} J$ Dermatol. 2013;168(3):474-485. doi:10.1111/bjd.12149 
33. Dreno B, Jean-Decoster C, Georgescu V. Profile of patients with mild-to-moderate acne in Europe: a survey. Eur $J$ Dermatol. 2016;26(2):177-184. doi:10.1684/ejd.2015.2722

34. Wei B, Pang Y, Zhu H, et al. The epidemiology of adolescent acne in North East China. J Eur Acad Dermatol Venereol. 2010;24(8):953957. doi:10.1111/j.1468-3083.2010.03590.x
35. Kosmadaki M, Katsambas A. Topical treatments for acne. Clin Dermatol. 2017;35(2):173-178. doi:10.1016/j.clindermatol.2016.10.010

36. Gupta M, Mahajan VK, Mehta KS, Chauhan PS. Zinc therapy in dermatology: a review. Dermatol Res Pract. 2014;2014:709152. doi:10.1155/2014/709152

\section{Publish your work in this journal}

Clinical, Cosmetic and Investigational Dermatology is an international, peer-reviewed, open access, online journal that focuses on the latest clinical and experimental research in all aspects of skin disease and cosmetic interventions. This journal is indexed on CAS
The manuscript management system is completely online and includes a very quick and fair peer-review system, which is all easy to use. Visit http://www.dovepress.com/testimonials.php to read real quotes from published authors. 